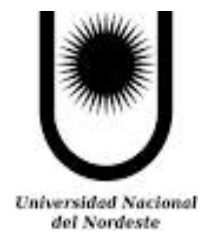

\author{
Facultad de Humanidades
}

UNIVERSIDAD NACIONAL DEL NORDESTE

Tesis Doctoral

\author{
LA MOVILIDAD COTIDIANA \\ EN RESISTENCIA, CHACO, ARGENTINA. \\ PROPUESTAS PARA \\ MICRO INTERVENCIONES \\ INTEGRALES URBANAS (NODOS)
}

Celmira Esther Rey

Año 2013

Publicado en formato digital: Dra. Celmira Esther REY. LA MOVILIDAD COTIDIANA EN RESISTENCIA, CHACO, ARGENTINA. PROPUESTAS PARA MICRO INTERVENCIONES INTEGRALES URBANAS (NODOS). RESÚMENES DE TESIS. Revista Geográfica Digital. IGUNNE. Facultad de Humanidades. UNNE. Año 13. № 25. Enero - Junio 2016. ISSN 1668-5180 Resistencia, Chaco.

En: http://hum.unne.edu.ar/revistas/geoweb/default.htm 


\section{La movilidad cotidiana en Resistencia, Chaco, Argentina. Propuestas para micro intervenciones integrales urbanas (nodos).}

Celmira Esther Rey

Directora: Dra. Ana María Foschiatti.

Co - Director: Lic. Rodolfo Valentín Bertoncello

Sumario

El presente trabajo ensaya identificar emergentes del funcionamiento de la movilidad cotidiana y su configuración territorial respectiva, en intersecciones o nodos de Resistencia, capital de la provincia del Chaco, Argentina. Específicamente, se evalúa el estado del equipamiento (infraestructura y mobiliario vial) de la vía pública y sobre modos o prácticas habituales de los sujetos usuarios de las mismas (peatones - pasajerosconductores de vehículos). Aspectos analizados teniendo en cuenta parámetros prescriptos en normativas vigentes y amparadas en los principios de accesibilidad, comunicación, equidad y justicia espacial que permitieron establecer un diagnóstico microespacial del estado en que se encuentran los nodos, objeto de estudio de esta tesis.

A partir del estado de situación detectado, sumado al producto obtenido de los discursos de los habitantes que habitualmente utilizan esas intersecciones, se postulan modelos de estado óptimo en los nodos seleccionados como escenarios territoriales accesibles y seguros en cuanto acondicionamiento físico material y, sobre modos correctos en que deben ser usados conforme las leyes que rigen a tal fin.

Los modelos resultantes se construyeron mediante el uso de programas informáticos con potencial suficiente para simular microambientes óptimos para circular. Esta última acción vislumbra, a través de la planificación territorial, proponer condiciones para que el desarrollo de la movilidad cotidiana se realice conforme una relación de complementaria entre usos, respeto entre las personas y el entorno inmediato.

Se plantea esta propuesta reconociendo que durante la movilidad se articulan complejas relaciones donde se pone de manifiesto el estilo de vida de una población, claramente identificable a través del acondicionamiento de la vía pública y el modo en que es utilizada por sus habitantes. De modo que, cada elemento componente del espacio analizado debe interactuar manteniendo un equilibrio dinámico tendiente a evitar conflictos y la potencial aparición de lesiones por esa causa.

Palabras claves

- Ambiente Urbano - Movilidad Cotidiana - Accesibilidad - Comunicación - equidadjusticia espacial- Normativas Vigentes - .

Publicado en formato digital: Dra. Celmira Esther REY. LA MOVILIDAD COTIDIANA EN RESISTENCIA, CHACO, ARGENTINA. PROPUESTAS PARA MICRO INTERVENCIONES INTEGRALES URBANAS (NODOS). RESÚMENES DE TESIS. Revista Geográfica Digital. IGUNNE. Facultad de Humanidades. UNNE. Año 13. № 25. Enero - Junio 2016. ISSN 1668-5180 Resistencia, Chaco.

En: http://hum.unne.edu.ar/revistas/geoweb/default.htm 


\section{The Daily Mobility in Resistencia, Chaco, Argentina.Proposals for micro comprehensive interventions urban (nodes).}

Celmira Esther Rey

Supervisors: Dra. Ana María Foschiatti at Lic. Rodolfo Valentín Bertoncello

Abstract

This work aims to identify emerging of the functioning of the daily mobility and its territorial configuration respective intersections or nodes of Resistencia, capital of the province of Chaco, Argentina. Specifically, it assesses the state of the equipment (equipment and infrastructure vial) of the public highway and on modes or practice of the subject users of the same (pedestrians, drivers of vehicles). Aspects analyzed taking into account parameters prescribed in regulations and covered in the principles of accessibility, communication and justice that space allowed to establish a diagnosis aggregated microespatial dispersion was observed in the state in which are the nodes, which are the subject of study of this thesis.

On the basis of the status detected, joined to the product obtained from the speeches of the inhabitants that regularly used these intersections, postulate models of optimal state in the selected nodes as spatial scenarios accessible and safe as fitness equipment and right ways in which they must be used in accordance with the laws that govern such order

The resulting models are constructed through the use of computer programs with sufficient potential to simulate microenvironments optimal for circular. This last action in sight, through the territorial planning, propose conditions for the development of-to-day mobility in accordance with a relationship between complementary uses, respect between people and the immediate environment.

It is raised by this proposal recognizing that during the mobility are articulated complex relationships where it reflects the lifestyle of a population, clearly identifiable through the fitting-out of the public highway and the way in which it is used by its inhabitants. In such a way that each component of the space analyzed must interact while maintaining a dynamic balance designed to avoid conflicts and the potential occurrence of injuries that cause.

Keywords:

Urban Environment - Daily Mobility - Accessibility - Communication - Equity - Justice space - Rules in force -

Publicado en formato digital: Dra. Celmira Esther REY. LA MOVILIDAD COTIDIANA EN RESISTENCIA, CHACO, ARGENTINA. PROPUESTAS PARA MICRO INTERVENCIONES INTEGRALES URBANAS (NODOS). RESÚMENES DE TESIS. Revista Geográfica Digital. IGUNNE. Facultad de Humanidades. UNNE. Año 13. № 25. Enero - Junio 2016. ISSN 1668-5180 Resistencia, Chaco.

En: http://hum.unne.edu.ar/revistas/geoweb/default.htm 


\section{Índice}

- Agradecimientos

- Resumen III

- Abstract IV

- Introducción 1

- Capítulo I: La movilidad cotidiana en el ámbito urbano 4

1. Presentación 4

2. Planteos preliminares 5

2.1. Pregunta central de investigación $\quad 5$

2.2. Planificar las intervenciones micro espaciales para optimizar la 6

$\begin{array}{ll}\text { 3. Objetivos } & 7\end{array}$

$\begin{array}{ll}\text { 3.1. Objetivos generales } & 7\end{array}$

3.2. Objetivos específicos $\quad 7$

4. La temática central de investigación: la movilidad cotidiana local 9

4.1. Configuración territorial de la estructura urbana 9

5. La vía pública y su incidencia en la movilidad cotidiana 10

5.1. La expresión de la vía pública 10

5.2. Peculiaridades de la movilidad cotidiana local 13

5.2.1. Derivaciones del desequilibrio ambiental en instancias de la 15 movilidad cotidiana

5.3. Las situaciones que generan conflicto en la movilidad cotidiana $\quad 17$

5.4. Aspectos generales de la situación de la movilidad cotidiana $\quad 19$

5.5. La situación a nivel mundial $\quad 19$

5.6 .La situación en Argentina 20

5.7. La situación en el Chaco 22

5.8.La situación en Resistencia 22

- Capítulo II: Las cuestiones teóricas y metodológicas para abordar un estudio sobre movilidad urbana $\quad 24$

1. Inserción de la movilidad cotidiana en los lineamientos epistemológicos $\quad 24$

contemporáneos: las bases teóricas 
1.1. Enfoques para potenciar estudios a nivel local y a micro escala territorial

1.1.1. Estudios de problemas contemporáneos a micro escala

1.2. La movilidad territorial como temática central de esta tesis

2. Estudios locales para extraer lecciones generales y soluciones a conflictos específicos

2.1. Los aportes del Grupo de estudio en Movilidad, Servicio,

Infraestructura y territorio (GEMSIT) a la investigación local

3. Las perspectivas metodológicas

3.1. Principales implicancias metodológicas

3.2. Metodologías adoptadas según procedencia de la información

3.3. Niveles de aproximación al objeto de estudio

3.3.1. Desde la mirada cuantitativa

3.3.2. Desde la mirada cualitativa

3.3.3. Desde la mirada de un método mixto complementario cuantitativo - cualitativo

3.4. La modelización de los nodos

3.5. Contribución de cada aporte metodológico

3.5.1. Aplicación de procedimientos propios de la metodología cuantitativa

3.5.1.1. Lineamientos generales del diseño de investigación cuantitativo

3.6. Pasos metodológicos

3.7. El área de estudio

3.7.1. Fundamentación de selección del área de estudio: los nodos elegidos y los corredores viales conectores

3.7.2. Criterios para la selección de las unidades de análisis: los nodos

3.8.Jerarquía de la estructura vial y su admisión jerárquica de movimientos

3.8.1. Jerarquía de movimientos

3.8.2. Zonificación de distritos

3.9. Relación de diferenciación territorial urbano: centro - periferia

3.10. Características socio demográficas del área de estudio

3.11. Identificación de áreas riesgosas

3.12. La información recolectada en los nodos. Dispositivos y etapas: definiciones conceptuales y metodológicas: captura, $\quad 76$ sistematización y tratamiento

3.13. Definición de criterios para la utilización de dispositivos de captura de datos in situ

3.14. Simulaciones que permiten demostrar condiciones óptimas a través de modelos de escenarios territoriales con situaciones reales de movilidad

4. El aporte cualitativo

5. Apreciaciones a modo de cierre: sugerencias para planificación de los 
nodos

- Capítulo III: El estado de los componentes de la movilidad cotidiana en 102 Resistencia, Chaco, en el contexto de la configuración territorial de la red vial

1. Presentación

2. El complejo funcionamiento de los componentes de la movilidad 104 cotidiana

2.1. La movilidad: un sistema complejo

105

2.2. Características de las interconexiones de la estructura vial

108

2.3. La conectividad de los nodos que enlazan los corredores viales en el área de estudio

2.4. La conectividad topológica como primera aproximación para comprender los nexos de la red vial.

2.4.1. La accesibilidad a los nodos a través de los corredoresconectores viales que los enlazan en el área de estudio

2.4.2. Barreras de carácter físico natural que obstaculizan la circulación vial

2.4.3. Barreras de carácter antrópico que se vinculan con la infraestructura vial y que obstaculizan la circulación vial

2.5. Los componentes de la movilidad. Acondicionamiento de la infraestructura y mobiliario vial

2.5.1. Los componentes materiales de la movilidad

2.5.2. Escasa infraestructura vial

2.5.3. Los componentes derivados de las acciones de los sujetos que se trasladan

2.6.La escena de la movilidad espacial en los nodos.

2.6.1. Las condiciones materiales de la vía pública

2.6.2. Los movimientos efectuados por los sujetos usuarios

3. El Diagnóstico en los Nodos seleccionados

3.1. Nodo 1: el microcentro

3.1.1. Recomendaciones de intervención en el Nodo $1 \quad 140$

3.2. Nodo 2: El límite del microcentro 142

3.2.1. Recomendaciones de intervención en el Nodo 2

3.3. Nodo 3: El límite del macrocentro 152

3.3.1. Recomendaciones de intervención en el Nodo 3

3.4. Nodo 4: El área periférica de la ciudad 161

3.4.1. Recomendaciones de intervención en el Nodo $4 \quad 180$

3.5. Nodo 5: Periferia y salida de la ciudad 181

3.5.1. Recomendaciones de intervención en el Nodo $5 \quad 188$

4. Consideraciones acerca del capítulo 188

- Capítulo IV: Propuestas de intervenciones integrales para optimizar la movilidad urbana

1. Presentación

Publicado en formato digital: Dra. Celmira Esther REY. LA MOVILIDAD COTIDIANA EN RESISTENCIA, CHACO, ARGENTINA. PROPUESTAS PARA MICRO INTERVENCIONES INTEGRALES URBANAS (NODOS). RESÚMENES DE TESIS. Revista Geográfica Digital. IGUNNE. Facultad de Humanidades. UNNE. Año 13. № 25. Enero - Junio 2016. ISSN 1668-5180 Resistencia, Chaco. 
2. Propuestas para planificación territorial referida a la movilidad de la población

3. Las propuestas y los alcances logrados con la participación de los observadores directos de los nodos y los decisores que intervienen en ellos

3.1. Remodelar y reacondicionar los nodos a partir de premisas de optimización de los escenarios territoriales deseados

3.2. Intervenciones prioritarias

4. Consideraciones aportadas por los observadores de los nodos

5. Estado de situación de la movilidad de la población

6. Principales emergentes de la percepción de los observadores de los nodos

7. Principales emergentes de las opiniones de los especialistas

8. Recomendaciones prevención micro intervenciones integrales de la movilidad cotidiana en nodos de Resistencia, Chaco, Argentina

235

Consideraciones finales para el cierre de esta investigación 242

Bibliografía

Anexo 1. Aportes personales en torno a la temática desarrollada

2.1.Experiencias vinculadas a lo educativo

2.2.Experiencias orientadas hacia la salud

Anexo 2. Aproximación conceptual

Anexo 3. Los planes estatales estratégicos vigentes

1.1.Articulación territorial, movilidad y su relación con la red vial de Resistencia, Chaco. Modelo actual y su proyección en diferentes escalas espaciales

1.2. La organización de la movilidad y estado de avance en la planificación territorial. Distintas escalas de análisis intersectoriales y espaciales

1.3.La planificación en la agenda gubernamental. Hacia una nueva configuración territorial

1.4.Plan Estratégico Nacional. Una visión en Red. Núcleos urbanos e Infraestructura de Transporte. Hacia un modelo policéntrico de ciudades

1.4.1. PET e Infraestructura de transporte

1.5.Plan Estratégico Provincial. La planificación en el ámbito provincial

1.6.La Planificación en el ámbito Municipal: Resistencia 2020

Anexo 4.Ampliaciones

1.7.Acuerdos entre Provincia y Municipios

Publicado en formato digital: Dra. Celmira Esther REY. LA MOVILIDAD COTIDIANA EN RESISTENCIA, CHACO, ARGENTINA. PROPUESTAS PARA MICRO INTERVENCIONES INTEGRALES URBANAS (NODOS). RESÚMENES DE TESIS. Revista Geográfica Digital. IGUNNE. Facultad de Humanidades. UNNE. Año 13. № 25. Enero - Junio 2016. ISSN 1668-5180 Resistencia, Chaco. 


\section{Índice de Esquemas, Imágenes, Mapas y Planos, Figuras, Tablas y Cuadros}

Págs.

\section{Esquemas}

$\mathrm{N}^{0} 1$ : La metodología aplicada 11

№2: La movilidad cotidiana en el ambiente urbano 12

$\mathrm{N}^{\circ}$ 4: Niveles de aproximación al objeto de estudio $\quad 42$

No5: Emergentes conceptuales a partir de los códigos hallados en los discursos $\quad 97$

№6: Grupo temático: La cuestión del género 98

$\begin{array}{ll}\mathrm{N}^{\circ} 7: \text { Los sujetos y la vía pública } & 102\end{array}$

No8: El complejo, dinámico y heterogéneo funcionamiento de la movilidad
cotidiana

No9:Movilidad cotidiana: interacción permanente entre seres humanos y

entorno

108

№10: Diagnóstico de la movilidad urbana

\section{Imágenes}

$\mathrm{N}^{\mathrm{o}} 1$ : Siniestro vial

$\mathrm{N}^{\circ}$ 2.1.: Aforo de conteo de sujetos usuarios de la vía

No2.2.: Aforo de conteo de sujetos usuarios de la vía 85

No3: Nodo 1: Desfase en la línea de rampas 133

$\mathrm{N}^{\circ}$ 4: Nodo 1: Influencia de la falta de alineación de rampas 134

No5: Nodo 1: Modificación del sentido de la circulación por presencia de
obstáculos

No6: Nodo 1: Presencia de propaganda política en señalización de la vía 136

Nº: Nodo 1: Presencia de obstáculos que interfieren en la movilidad 137

No8: Nodo 1: Prolongación comercial en la vereda 138

$\begin{array}{ll}\mathrm{N}^{\circ} \text { 9: Nodo 2: Parada de transporte público } & 144\end{array}$

$\begin{array}{ll}\mathrm{N}^{\circ} 10: \text { Nodo 2: Presencia de canteros en esquina } & 144\end{array}$

No10 (bis): Nodo 2: Presencia de canteros en esquina 149

No11: Nodo 2: Presencia de obstáculos en ochava 145

No11 (bis): Nodo 2: Presencia de obstáculos en ochava 145

No12: Nodo 2: Ubicación y estado de rampa 146

$\begin{array}{ll}\mathrm{N}^{\circ} 13: \text { Nodo 2: Sitio de fricción } & 148\end{array}$

No14: Nodo 2: Rampa en mal estado 151

No15: Vista del Nodo $3 \quad 153$

No16: Vista del Nodo $3 \quad 154$

№17: Nodo 3: Estado de las veredas en esquina 155

No18: Nodo 3: Esquina A 155

No19: Nodo 3: Situación de la ochava en esquina C 158

Publicado en formato digital: Dra. Celmira Esther REY. LA MOVILIDAD COTIDIANA EN RESISTENCIA, CHACO, ARGENTINA. PROPUESTAS PARA MICRO INTERVENCIONES INTEGRALES URBANAS (NODOS). RESÚMENES DE TESIS. Revista Geográfica Digital. IGUNNE. Facultad de Humanidades. UNNE. Año 13. № 25. Enero - Junio 2016. ISSN 1668-5180 Resistencia, Chaco.

En: http://hum.unne.edu.ar/revistas/geoweb/default.htm 
$\mathrm{N}^{\circ}$ 20: Nodo 3: Esquina B

$\mathrm{N}^{\circ} 21$ : Nodo 4: Vista aérea

No22: Nodo 4: Áreas de adyacencia

163

$\mathrm{N}^{\circ} 23$ : Nodo 4: Artesano de la etnia Toba

$\mathrm{N}^{\circ}$ 24: Nodo 4: Variedad de medios de transporte

$\mathrm{N}^{\circ}$ 25: Nodo 4: Sujetos usuarios

$\mathrm{N}^{\circ}$ 26: Nodo 4: Presencia de guarda rail

No27: Nodo 4: Las condiciones materiales de la vía pública

$\mathrm{N}^{\circ}$ 28: Nodo 4: Las condiciones materiales de la vía pública

No29: Nodo 4: Insuficiente señalización vial

$\mathrm{N}^{\circ} 30$ : Nodo 4: Presencia de guarda rail

No31: Nodo 4: Desniveles en calzada

173

$\mathrm{N}^{\circ} 32$ : Nodo 4: Estado de la infraestructura

No33: Nodo 4: Situación de la señalización vial 174

No34: Nodo 4: Senda peatonal

No35: Nodo 4: Secuencia cruce peatonal 176

No36: Nodo 4: Estado de la esquina 178

No37: Nodo 5: Vista del Nodo $5 \quad 182$

№38: Nodo 5: Vista aérea de la intersección y sus inmediaciones 183

No39: Nodo 5: Esquina A 184

$\mathrm{N}^{\circ}$ 40: Nodo 5: Dificultades en el cruce peatonal 185

$\mathrm{N}^{\circ}$ 41: Nodo 5: Avenida 25 de Mayo y sus ramificaciones con orientaciones 187

notoriamente diferenciadas

N 42: Nodo 5: Incorrecta circulación de motociclistas

\section{Mapas y planos}

$\mathrm{N}^{\circ}$ 1: Ejes de integración y desarrollo (en Anexo 3)

№2: Plan Estratégico Territorial Provincial deseado, 2008(en Anexo 3).

$\mathrm{N}^{\circ}$ 3: Plan Estratégico Territorial de la provincia del Chaco: Obras prioritarias deseadas, 2008 (en Anexo 3).

No4: Plan Estratégico territorial de la Provincia del Chaco: Áreas prioritarias, 2011(en Anexo 3)

$\mathrm{N}^{\circ} 5$ : Red vial de la Provincia del Chaco (en Anexo 3).

$\mathrm{N}^{\circ}$ 6: Resistencia: Plan director de obras viales (en Anexo 3)

$\mathrm{N}^{\circ}$ 7: El área de estudio dentro de la trama vial de Resistencia

No8: Jerarquía de la Red Urbana en Resistencia

$\mathrm{N}^{\circ}$ 9: Resistencia. Zonificación de Distritos

$\mathrm{N}^{0}$ 10: Densidad de la población por zona

Nº11: Niveles socioeconómicos en el Gran Resistencia. Año 2001. Estrato predominante por manzana.

Nº12: Áreas de mayor riesgo. 2000. Áreas observadas.

N¹3: El sentido de circulación en las vías de la ciudad de Resistencia $\quad 69$

№14: Sentido de Circulación Homologado en Resistencia 111

№15: Conectividad Topológica

Publicado en formato digital: Dra. Celmira Esther REY. LA MOVILIDAD COTIDIANA EN RESISTENCIA, CHACO, ARGENTINA. PROPUESTAS PARA MICRO INTERVENCIONES INTEGRALES URBANAS (NODOS). RESÚMENES DE TESIS. Revista Geográfica Digital. IGUNNE. Facultad de Humanidades. UNNE. Año 13. № 25. Enero - Junio 2016. ISSN 1668-5180 Resistencia, Chaco.

En: http://hum.unne.edu.ar/revistas/geoweb/default.htm 
$\mathrm{N}^{\circ}$ 16: La ciudad de Resistencia

№17: Barreras que interfieren en el sentido de la circulación vial

$\mathrm{N}^{\mathrm{0}}$ 18: Resistencia: Isocronas en corredores que enlazan nodos seleccionados.

Año 2012

N¹9: Evolución de la Ocupación urbana AMGR

No20: Pavimentos AMGR - 1998

№21: Ubicación Nodo 1

No22: Ubicación Nodo 2

N²3: Ubicación Nodo 3

\section{Figuras:}

$\mathrm{N}^{\circ} 1$ : Nodo de articulación territorial interprovincial, nacional e internacional (en Anexo 3)

No2: Esquema policéntrico de ciudades (en Anexo 3)

Nº3: Presentación de obra de Autovía Ruta Nacional №11 (en Anexo 3)

№4: Perfil transversal de la Autovía Ruta Nacional Nº11-travesía urbana (en Anexo 3)

№5: Perfil en planta de la Autovía Ruta Nacional №11- travesía urbana- (en Anexo 3)

No6: Autopista: Segunda etapa de la Autovía Ruta Nacional Nº11 -travesía urbana- (en Anexo 3)

N7: Propuestas vigentes referidas a la planificación territorial que incumbe a la movilidad cotidiana analizada (en Anexo 3).

No8: Componentes de la vía Pública

$\mathrm{N}^{\circ}$ 9: Jerarquía de los movimientos

$\mathrm{N}^{\circ} 10$ : Ubicación del observador

No11: Recorrido dentro del nodo para la captura de información 81

$\mathrm{N}^{\circ} 12$ : Tiempo de recorrido en circuito $\mathrm{A}$

$\mathrm{N}^{\mathrm{o}}$ 13: Tiempo de recorrido en circuito B

$\mathrm{N}^{\circ} 14$ : Infraestructura del Nodo 1

No15: Nodo 2: Puntos de fricción en calzadas 147

No16: Dimensiones de las veredas 150

No17: Nodo 3: Infraestructura 153

No18: Nodo 4: Dimensiones de las calzadas 162

№19: Gráfico de sujetos usuarios: Ingreso Nodo 4

No20: Nodo 4: Infraestructura y Uso del suelo 167

No21: Nodo 4: Infraestructura deficiente 177

No22: Nodo 4: Sentido de la circulación 179

No23: Nodo 4: Inmediaciones $\quad 180$

No24: Nodo 1: Esquema en perspectiva de calle Arturo Illia hacia calles $\quad 194$

descendentes

195

$\mathrm{N}^{\circ} 25$ : Nodo 1: Esquema en planta

Publicado en formato digital: Dra. Celmira Esther REY. LA MOVILIDAD COTIDIANA EN RESISTENCIA, CHACO, ARGENTINA. PROPUESTAS PARA MICRO INTERVENCIONES INTEGRALES URBANAS (NODOS). RESÚMENES DE TESIS. Revista Geográfica Digital. IGUNNE. Facultad de Humanidades. UNNE. Año 13. № 25. Enero - Junio 2016. ISSN 1668-5180 Resistencia, Chaco.

En: http://hum.unne.edu.ar/revistas/geoweb/default.htm 
N²6: Nodo 1: Esquema en perspectiva de calle Arturo Illia hacia calles ascendentes

$\mathrm{N}^{\circ} 27$ : Nodo 2: Esquema en planta

№28: Nodo 2: Esquema en perspectiva de la intersección calle Santiago del Estero y Avenida Belgrano. Proyectado calle Santiago del Estero hacia los

descendentes

N²9: Nodo 3: Sentido de la circulación

$\mathrm{N}^{\circ}$ 30: Nodo 3: Vista en planta

No31: Nodo 4: Propuesta de reformulación 206

No32: Nodo 5: propuesta de reformulación 207

No33: Nodo 5: Sentido de la circulación 208

No34: Nodo 5: Vista en perspectiva 208

No35: Nodo 5: Estado en el año 1998

No36: Nodo 5: Estado actual del nodo 224

\section{Tablas y cuadros:}

Nº1: Características típicas del sistema urbano vial 58

No2: Variables e indicadores utilizados para el análisis de la movilidad $\quad 74$

cotidiana urbana

№3: Planilla de observación de las condiciones de la Vía Pública 76

$\begin{array}{ll}N^{\circ} 4: \text { Matriz de datos } & 93\end{array}$

No5: Dimensiones analíticas vertical y horizontal 95

Nº6: Proceso de codificación organizado a partir de códigos derivados del 96

marco conceptual y de códigos emergentes en el discurso de cada entrevistado

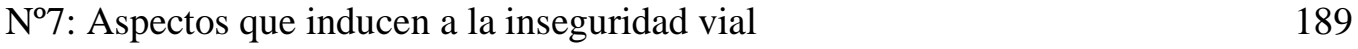

No8: Niveles de intervenciones prioritarias 210

$\mathrm{N}^{\circ}$ 9: Intervenciones prioritarias en la movilidad en los nodos seleccionados $\quad 210$

$\mathrm{N}^{\circ} 10$ : Sugerencias de los observadores directos para mejorar la seguridad vial en los nodos 


\section{Introducción}

Reconociendo que la movilidad cotidiana en Resistencia, Chaco, se desenvuelve en un marco geográfico donde el contexto general muestra una tendencia a la pérdida de solidaridad, desprotección, falta de acción tutelar efectiva e incumplimiento de las normativas vigentes, se cree conveniente exhortar a las autoridades para que la toma de decisiones esté focalizada en políticas públicas locales que apunten hacia una organización espacial planificada y en función de las problemáticas detectadas. (Rey, 2008). En términos generales, esa fue la situación diagnosticada a fines de los años 90 , y expuesta en el año 2001 en instancias de la elaboración personal de la Tesis de Maestría con el título: “Las condiciones ambientales de la vida urbana. El tránsito como generador de riesgo de accidentes en la ciudad de Resistencia. Facultad de Arquitectura y Urbanismo, UNNE.

Transcurrido el tiempo desde aquel escrito y fruto de los cursos de Postgrados, dentro de la Carrera de Doctorado en Geografía de la Facultad de Humanidades, UNNE, se logró producir una serie de investigaciones mediante la incorporación de innovadores aportes teóricos, la utilización de procedimientos metodológicos de vanguardia y el apoyo de los avances tecnológicos propios de la Geografía (entre ellos, los SIG). 
Teniendo en cuenta lo precedentemente expuesto y, como miembro componente del Instituto de Geografía de la Facultad de Humanidades, UNNE, es oportuno señalar la incorporación de estudios con enfoques referidos a vulnerabilidad y planificación territorial, en esta tesis vinculados con las condiciones ambientales de la movilidad cotidiana urbana, específicamente, en particular las que inducen a ocasionar conflictos en la vía pública, y generan situaciones de riesgo psicofísicas en la población durante su circulación en la ciudad de Resistencia, capital de la Provincia del Chaco, Argentina.

Las consideraciones que se contemplan en los capítulos del cuerpo de este trabajo escrito responden a:

- Circunscribir la temática en el contexto actual: Capítulo I: “La movilidad cotidiana en el ámbito urbano” (Abarca 23 páginas).

- Combinar instrumentos analíticos, algunos de los cuales fueron adoptados, otros adaptados, creados y puestos a prueba en la tesis de Maestría (2001), pero ahora con el aporte de la metodología cualitativa y la incorporación de otras herramientas como la utilización de los SIG, tal como se expone en el Capítulo II: "Las cuestiones teóricas y metodológicas para abordar un estudio sobre movilidad urbana" (Comprende 80 páginas donde se justifican y fundamentan procedimientos para la captura de datos y su tratamiento, bajo resguardo de criterios de confiabilidad y validez).

- Reflejar las condiciones de la movilidad cotidiana en Resistencia, en el marco de su complejidad. Vale decir, el “como” se produce la movilidad local en los nodos seleccionados, dando cuenta de los hechos desencadenantes producto de la intrincada trama de interrelaciones que se producen en cada uno de micro territorios urbanos estudiados: Capítulo III. "El estado de sus componentes de la movilidad cotidiana en Resistencia, Chaco, en el contexto de la Configuración territorial de la red vial urbana” (Abarca 89 páginas).

Por último, en el Capítulo IV: "Propuestas de intervenciones integrales para optimizar la movilidad urbana” (de 45 páginas), se exponen lineamientos sugerentes para una planificación integral de los nodos, amparadas en principios directrices como accesibilidad, comunicación, equidad, justicia espacial y la participación ciudadana.

Publicado en formato digital: Dra. Celmira Esther REY. LA MOVILIDAD COTIDIANA EN RESISTENCIA, CHACO, ARGENTINA. PROPUESTAS PARA MICRO INTERVENCIONES INTEGRALES URBANAS (NODOS). RESÚMENES DE TESIS. Revista Geográfica Digital. IGUNNE. Facultad de Humanidades. UNNE. Año 13. № 25. Enero - Junio 2016. ISSN 1668-5180 Resistencia, Chaco.

En: http://hum.unne.edu.ar/revistas/geoweb/default.htm 
Esto último, como producto de una acción colaborativa de actores sociales usuarios de la vía pública , los referentes sociales y los funcionarios gubernamentales, atendiendo a una concepción más acabada de lo que significa la construcción del conocimiento en ámbitos académicos de gestión pública estatal.

Se diagnosticó el estado de situación de intersecciones (nodos) seleccionadas de la red vial, centrando el análisis en fundamentos rectores vinculados con las normativas vigentes, la accesibilidad, la comunicación, la equidad y la justicia territorial de los entornos objeto de análisis, así como de las personas que hacen uso de esos lugares. Como propuesta de planificación se presentan modelos de estado óptimo de los nodos, demostrando en qué medida, lo diagnosticado se acerca o se aleja de principios rectores enunciados con anterioridad. Es prioritario que los responsables de la administración y gestión del territorio, junto con los referentes comunitarios, garanticen a los ciudadanos la máxima seguridad posible durante su movilidad en la vía pública. En contraparte, se espera que los ciudadanos, respeten las normas, puesto que en ellas subyacen medidas de protección para sí y para con el otro. De esta manera, trabajando mancomunadamente se contribuiría a producir los cambios necesarios para una convivencia pacífica en un ambiente equilibrado y armónico, propendiendo a mejorar la calidad de vida de todos sus habitantes.

\section{Planificar las intervenciones micro espaciales para optimizar la movilidad cotidiana}

Los alcances logrados dan un estado de diagnóstico de las condiciones físico materiales en distintos sitios de la ciudad de Resistencia, producto de la combinación de la multiplicidad de factores intervinientes, tales como los componentes del estado de la seguridad vial, las acciones humanas durante los desplazamientos cotidianos y las peculiaridades del entorno físico material de la red vial. A la vez, se delinean medidas de mitigación tendientes a la prevención de los riesgos en la vía pública que podrían ser de interés municipal, como para el área educativa y de salud provincial, intentando de este modo, contribuir al desarrollo local de la comunidad. Esta última medida, a partir de la participación ciudadana usuarios de la vía pública y de los planificadores. Por

Publicado en formato digital: Dra. Celmira Esther REY. LA MOVILIDAD COTIDIANA EN RESISTENCIA, CHACO, ARGENTINA. PROPUESTAS PARA MICRO INTERVENCIONES INTEGRALES URBANAS (NODOS). RESÚMENES DE TESIS. Revista Geográfica Digital. IGUNNE. Facultad de Humanidades. UNNE. Año 13. № 25. Enero - Junio 2016. ISSN 1668-5180 Resistencia, Chaco.

En: http://hum.unne.edu.ar/revistas/geoweb/default.htm 
último, se esbozan propuestas sobre líneas prioritarias para planificar las intervenciones en los nodos, con el objeto de optimizar la movilidad cotidiana en la ciudad de Resistencia. Desde una perspectiva integral, se presentan escenarios territoriales deseados a partir de la situación actual de nodos seleccionados a tal fin, partiendo desde el micro centro, pasando por el macro centro ${ }^{1}$, hasta llegar al área periférica Oeste de la ciudad de Resistencia.

A partir de los objetivos generales propuestos:

1. Se diagnosticó el estado actual de la movilidad cotidiana en sitios seleccionados de Resistencia sobre parámetros avalados en principios directrices (accesibilidad al medio físico material de la vía pública por parte de los sujetos usuarios de la misma, equipamiento referidos a la comunicación , equidad, justicia territorial y seguridad vial).

2. Se logró recrear modelos de optimización, referidos a las condiciones físicas materiales y de las acciones manifiestas de los sujetos usuarios en aquellos sitios seleccionados de la ciudad de Resistencia, teniendo en cuenta parámetros precedentemente enunciados.

3. Se tradujeron los emergentes socioculturales ${ }^{2}$ que permitieron brindar lineamientos relacionados con la planificación de los nodos, como una puesta en práctica de una acción colaborativa y solidaria entre ciudadanos y decisores. Acción estratégica orientada a fomentar la participación ciudadana conjunta para el logro de un desarrollo local equilibrado, armonioso y de carácter democrático.

Se brinda una serie de propuestas de mitigación para los nodos (intersecciones de la red vial urbana) en dos sentidos, una orientada a la planificación, ordenamiento y organización territorial y otra, destinada al ámbito educativo y/o salud, como herramientas para iniciar un proceso de concientización sobre educación para la seguridad vial, propendiendo a una óptima sustentabilidad ambiental urbana local.

1Como generadores de los principales flujos de movilidad, donde se evidencian áreas cada vez más alejadas y distantes, tanto en distancias reales, como en equipamiento, en servicios, en definitiva en accesibilidad y seguridad vial.

2 Teniendo en cuenta la opinión de la población entrevistada.

Publicado en formato digital: Dra. Celmira Esther REY. LA MOVILIDAD COTIDIANA EN RESISTENCIA, CHACO, ARGENTINA. PROPUESTAS PARA MICRO INTERVENCIONES INTEGRALES URBANAS (NODOS). RESÚMENES DE TESIS. Revista Geográfica Digital. IGUNNE. Facultad de Humanidades. UNNE. Año 13. № 25. Enero - Junio 2016. ISSN 1668-5180 Resistencia, Chaco.

En: http://hum.unne.edu.ar/revistas/geoweb/default.htm 
Revista Geográfica Digital. IGUNNE. Facultad de Humanidades. UNNE. Año 13. № 25.

Enero - Junio 2016. ISSN 1668-5180 Resistencia, Chaco

Celmira Esther Rey

Publicado en formato digital: Dra. Celmira Esther REY. LA MOVILIDAD COTIDIANA EN RESISTENCIA, CHACO, ARGENTINA. PROPUESTAS PARA MICRO INTERVENCIONES INTEGRALES URBANAS (NODOS). RESÚMENES DE TESIS. Revista Geográfica Digital. IGUNNE. Facultad de Humanidades. UNNE. Año 13. № 25. Enero - Junio 2016. ISSN 1668-5180 Resistencia, Chaco.

En: http://hum.unne.edu.ar/revistas/geoweb/default.htm 